\title{
Evodiamine Selectively Inhibits Multiple Myeloma Cell Growth by Triggering Activation of Intrinsic Apoptosis Pathway
}

This article was published in the following Dove Press journal: OncoTargets and Therapy

\section{Qing Fang (D* \\ Siyi Jiang (iD* \\ Chengyuan $\mathrm{Li}$}

Department of Hematology, The Third Xiangya Hospital of Central South University, Changsha, People's Republic of China

*These authors contributed equally to this work
Correspondence: Chengyuan Li The Third Xiangya Hospital of Central South University, Tongzipo Road I38, Changsha 410013, People's Republic of China

Tel/Fax +86073I886I85II

Email Icy800097@I63.com
Introduction: Evodiamine (Evo) is one of the main bioactive components derived from the drying mature fruit of the genus Evodia rutaecarpa (Juss.) Benth. Although Evo has shown its anti-cancer activity in several cancers, the effects on multiple myeloma (MM) remain unknown. In this study, we aim to investigate the cytotoxic role of Evo on MM cells.

Methods: CCK- 8 assay, apoptotic cell analysis, xenografted mice model, caspase activity assay and mitochondrial membrane potential assay were performed.

Results: We found that Evo selectively inhibits cell proliferation and increases apoptosis rate in MM cells, but not in healthy B lymphocytes, in a time and dose-dependent manner. Evo treatment significantly activated caspase- 3 and -9 in MM cells. Evo also increased cytochrome $\mathrm{C}$ expression and ROS production in cytosol in a dose-dependent manner, which was abolished by MitoTEMPO cotreatment. In addition, co-treatment with bortezomib and Evo showed a more potent reduction of cell viability and a higher apoptosis than that of bortezomib single treatment in U266 and RPMI8226 cells.

Conclusion: We provided evidence to demonstrate that Evo selectively suppresses cell growth and increases apoptosis rate in MM cells through the intrinsic apoptosis pathway. Application of Evo and bortezomib might enhance the anti-cancer effect on MM cells.

Keywords: evodiamine, mitochondria, intrinsic apoptosis, bortezomib, resistance

\section{Introduction}

Multiple myeloma (MM) is a common hematologic malignancy that rises from cancerous B cells. Unlimited proliferation is the main characteristic of MM cells. ${ }^{1} \mathrm{MM}$ cells continuously produce various antibodies and pro-inflammatory factors, resulting in severe symptoms. ${ }^{2}$ Recently, bortezomib (BTZ), a proteasome inhibitor, treatment has greatly increased the free survival and overall survival of patients with MM. ${ }^{3}$ However, intrinsic and acquired resistance to BTZ would occur after several cycles' treatment. Therefore, a new effective drug is urgently required to overcome BTZ resistance. ${ }^{4}$

The bioactive substances from traditional Chinese medical herbs have great therapeutic effects in anti-cancer. ${ }^{5}$ Evodiamine (Evo) is one of the main bioactive components derived from the drying mature fruit of the genus Evodia rutaecarpa (Juss.) Benth., which is a traditional Chinese medicine with a hundred years of history. ${ }^{6}$ Evodiamine has shown anti-cancer activity in several cancers. Evo can induce apoptosis of human hepatocellular carcinoma HepG2, melanoma A375-S2, cervical cancer HeLa and other tumor cells. ${ }^{7-9}$ In addition, Evo can cause nuclear translocation factor NF- $\mathrm{B}$ translocation in A375-S2 cells, and suppress PI3K/AKT 
and activating MAPK in glioma, as well as affect the expression of microRNAs. ${ }^{10-13}$ After pretreatment with ubiquitin-proteasome inhibitor MG132, evodiamine caused more A375-S2 cell death. MG132 can increase activate caspase-3 and down-regulate the expression of Bcl-2, suggesting that evodiamine is involved in caspaseinduced apoptosis, which can be enhanced by the ubiquitin-proteasome pathway. ${ }^{14}$ However, the effects and underlying mechanism of Evo on MM remain unknown.

In the present study, we aim to test the cytotoxic role and underlying mechanism of Evo on MM cells. We provided evidence to demonstrate that Evo selectively inhibits cell proliferation and increases apoptosis rate in MM cells through ROS accumulation.

\section{Materials and Methods}

\section{Cell Lines Culture}

U266 and RPMI8226 were purchased from ATCC (Manassas, USA). Periphery B lymphocytes (HBC) were isolated from peripheral blood donated by healthy individuals of the Third Xiangya Hospital using a human Pan B Cell Isolation Kit (cat no. 130-101-638, Miltenyi Biotec, Somerville, MA, USA) following manufacturer's instructions. All cells were cultured in RPMI-1640 medium supplemented with 10\% FBS (fetal bovine serum) (Thermo Fisher Scientific). The blood donors have provided written informed consent. This study was approved by the Ethical Review committee of the Third Xiangya Hospital, Central South University (Changsha, China).

\section{Cell Treatment}

U266 and RPMI8226 cells treated with Evo (50, 100, 200, $400,800 \mu \mathrm{g} / \mathrm{mL}$ ) for $24 \mathrm{~h}$ or treated with $400 \mu \mathrm{g} / \mathrm{mL}$ Evo for different time points $(12,24,36,48 \mathrm{~h})$. To evaluate the effects of Evo on bortezomib-induced cell apoptosis and proliferative inhibition, the cells were co-treated with 40 nM bortezomib (cat no. S1013, Selleck, Shanghai, China) for $24 \mathrm{~h}$, and then were plated in 96-well plates at a density of 1000 cells in $100 \mu \mathrm{L}$ of the aforementioned RPMI-1640+FBS media per well at $37^{\circ} \mathrm{C}$ with $5 \% \mathrm{CO}_{2}$.

\section{Measurement of Cell Viability}

A Cell Counting Kit-8 (Abbkine, USA) was used to measure cell viability after indicated treatment following the manufacturer's instructions. Briefly, 2000 cells were seeded and cultured for $24 \mathrm{~h}$ and treated with Evo with or without bortezomib for $24 \mathrm{~h}$. Following further incubation for different time points
(12, 24, 36, $48 \mathrm{~h}), 10 \mu \mathrm{L}$ CCK-8 reagents were added to each well. OD value was measured by a microplate reader.

\section{Apoptosis Assays}

TiterTACS In Situ Detection Kit, a TUNEL-based assay (Catalog\#4822-96-K, Trevigen, USA) was used to measure cell apoptosis rate in vitro following the manufacturer's protocol. Briefly, cells were collected after various treatments and then resuspended in Buffered Formaldehyde at $1 \times 10^{6}$ cells/ $\mathrm{mL}$ for $7 \mathrm{~min}$. The cells were washed and incubated with a TACS-Sapphire substrate for $30 \mathrm{~min}$. The TACS 2 TdT$\mathrm{DAB}$ was used to measure the percentage of apoptotic cells.

\section{Xenograft Model and Treatment}

$\mathrm{BALB} / \mathrm{C}$ nude mice (6-week-old, male) were purchased from the Animal center of Central South University (Changsha, China). Animal experiments were approved by the Ethical Review committee of the Third Xiangya Hospital following the National Research Council's Guide for the Care and Use of Laboratory Animals (Eight Edition). About $2 \times 10^{5}$ of U266 cells were subcutaneously injected into a mouse. When the tumor volume reached about $150 \mathrm{~mm}^{3}$, the mice were injected with Evo $(100 \mathrm{mg} / \mathrm{kg})$ or bortezomib $(50 \mathrm{mg} / \mathrm{kg})$ by tail vein every 2 days for three times. Bodyweight and xenograft volume were monitored every 2 days. The tumor sizes were measured regularly and calculated using the following formula: $0.5 \times \mathrm{L} \times \mathrm{W}^{2}$, where $\mathrm{L}$ and $\mathrm{W}$ refer to the length and width of the tumor, respectively. The xenograft tumor tissues were obtained at day 20 for further analysis.

\section{Caspase Activity Assay}

Caspase 3, 8, 9 Activity Assay Kits (cat no. C1108, C1151, and C1158) were purchased from Beyotime Inc. (Shanghai, China). They were used to measure the activity of caspase$3,-8$ and -9 according to the manufacturer's protocol.

\section{Western Blot}

The tissues or cells were lysed in RIPA buffer. Antibodies for detecting Bax (cat no. 5023, 1:1000, Cell Signaling Technology), Bcl2 (cat no. 15071, 1:1000, Cell Signaling Technology), active Caspase-3 (cat no. 9664, 1:1000, Cell Signaling Technology), MCL-1 (cat no. 94296, 1:1000, Cell Signaling Technology), and GAPDH (cat no. 5174, 1:2000, Cell Signaling Technology) were applied according to the manufacturer's protocol. A cytochrome c release assay kit (GeneTex, Irvine, CA, USA) was used for measuring cytochrome $\mathrm{C}$ expression according to the manufacturer's protocol. Cells were homogenized and separated into cytosol 
and mitochondria extraction. Western blotting was performed to detect the release of Cytochrome $\mathrm{c}$ by using the cytochrome c antibody provided in the kit. Semi-quantification of Western blot bands was analyzed using Image $\mathrm{J}$ software.

\section{Mitochondrial Membrane Potential Assay}

A tetramethylrhodamine ethyl ester (TMRE)-mitochondrial membrane potential assay kit (cat no. 701310, Ann Arbor, Michigan, USA) was used for Mitochondrial membrane potential detection according to the manufacturer's protocol. Cells were incubated with $100 \mu \mathrm{M}$ TMRE working solution at $37^{\circ} \mathrm{C}$ for $30 \mathrm{~min}$. TMRE staining was analyzed using a fluorescence plate reader $($ Excitation/Emission=530/580 nm).

\section{Reactive Oxygen Species (ROS) Measurement}

A reactive oxygen species assay kit (Cat No. A057, FulenGen Co., Ltd, Guangzhou, China) was used for cellular ROS level detection according to the manufacturer's protocol.

\section{Statistical Analysis}

Data were expressed as mean \pm SD. Graphpad Prism Software (Ver.8) was used for data analysis. Multiple comparison was performed using one-way analysis of variance with post hoc Tukey's test. $\mathrm{p}<0.05$ is considered as significance.

\section{Results}

\section{Evo Treatment Inhibits Cell Growth in MM Cells}

Two MM cell lines (U266 and RPMI8226) and HBC were used to investigate the role of Evo on MM cells. Various concentrations of Evo were added into the culture medium and incubated for $24 \mathrm{~h}$, and then the cell viability was detected. Evo treatment downregulated U266 and RPMI8226 cell viability in a dose-dependent manner. There were no significant effects on HBC after Evo treatment (Figure 1A). Additionally, treatment of Evo $(400 \mu \mathrm{g} / \mathrm{mL})$ inhibited cell viability of U266 and RPMI8226 cells in a time-dependent manner, and Evo treatment did not influence the viability of HBC (Figure 1B). Evo also dramatically increased cell apoptosis rate in U266 and RPMI8226 cells in time and dose-dependent manner (Figure 1C).

Furthermore, we found that Evo treatment $(400 \mathrm{mg} / \mathrm{kg})$ significantly suppressed tumor growth in vivo (Figure 1D); however, Evo treatment did not affect the xenografted mice body weight, suggesting that Evo treatment had no significant toxic to these mice (Figure 1E). TUNEL expression was significantly increased (Figure 1F). In addition, the Bax expression was increased, while $\mathrm{Bcl} 2$ expression was reduced by Evo treatment in tumor tissues (Figure 1G), indicating that Evo treatment leads to a significant increase in cell apoptosis in xenograft tumor. These evidences indicate that Evo treatment could inhibit MM cell growth in vitro and in vivo.

\section{Evo Treatment Inhibits MM Cell Growth by Activating the Intrinsic Mitochondria Apoptosis Pathway}

To determine whether Evo induced apoptosis is associated with intrinsic apoptosis pathway, we treated U266 and RPMI8226 cells with different concentrations of Evo for $24 \mathrm{~h}$ and measured the caspase- $3,-8$ and -9 activity. Our results demonstrated that Evo treatment significantly activated caspase- 3 and -9 , while only moderate influence on caspase- 8 activity when Evo treatment at a high concentration (Figure 2A-C). In addition, Evo treatment dramatically increased the expression of active caspase 3 and Bax, while reduced the expression of $\mathrm{Bcl} 2$, suggesting a significant increase in cell apoptosis (Figure 2D and E).

Furthermore, we determined the release of Cytochrome $\mathrm{C}$ from mitochondria to cytosol. We found that Evo treatment upregulated cytochrome $\mathrm{C}$ levels in cytosol and decreased its levels in mitochondria in U266 and RPMI8226 cells in a dose-dependent manner (Figure 3A-C), suggesting that Evo treatment increased the release of mitochondrial cytochrome $\mathrm{C}$ from mitochondria into cytosol. In addition, we observed that Evo treatment also accordingly decreased TMRE in U266 and RPMI8226 cells (Figure 3D).

We next detected general ROS production, which could activate intrinsic mitochondria apoptosis pathway, in MM cells after Evo treatment. We found that Evo treatment dramatically upregulated cell apoptosis and ROS levels in U266 and RPMI8226 cells, which can be significantly abolished by MitoTEMPO cotreatment (Figure 3E and F). These results suggest that the cytotoxicity of Evo was associated with mitochondrial superoxide production. Thus, we inferred that Evo treatment selectively induces MM cells' apoptosis but not HBC through intrinsic apoptosis pathway by accumulating ROS production.

\section{Evo Enhances Bortezomib Effects on Multiple Myeloma}

Required resistance to bortezomib in MM cells may result in relapse. We next tested whether Evo could synergize with bortezomib in MM cells. We treated U266 and RPMI8226 

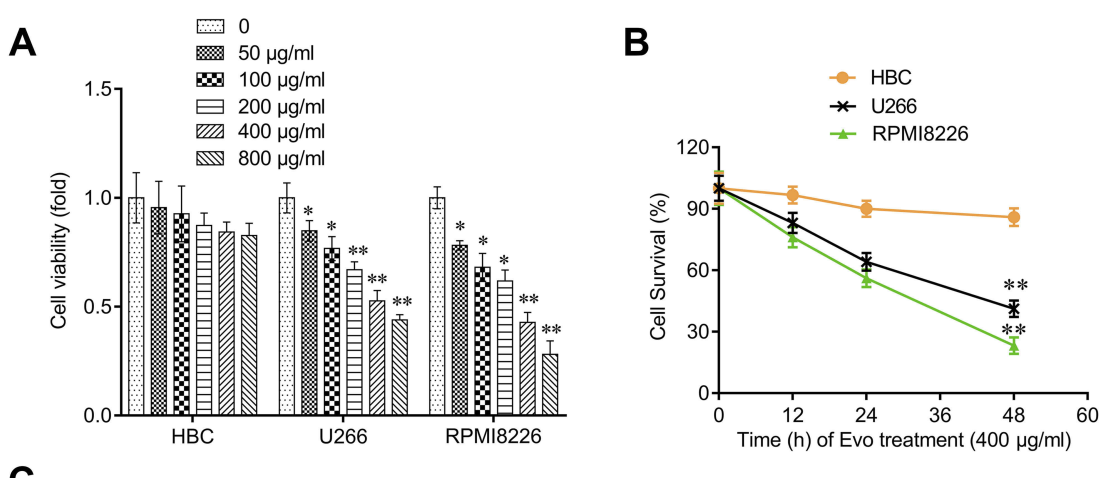

C
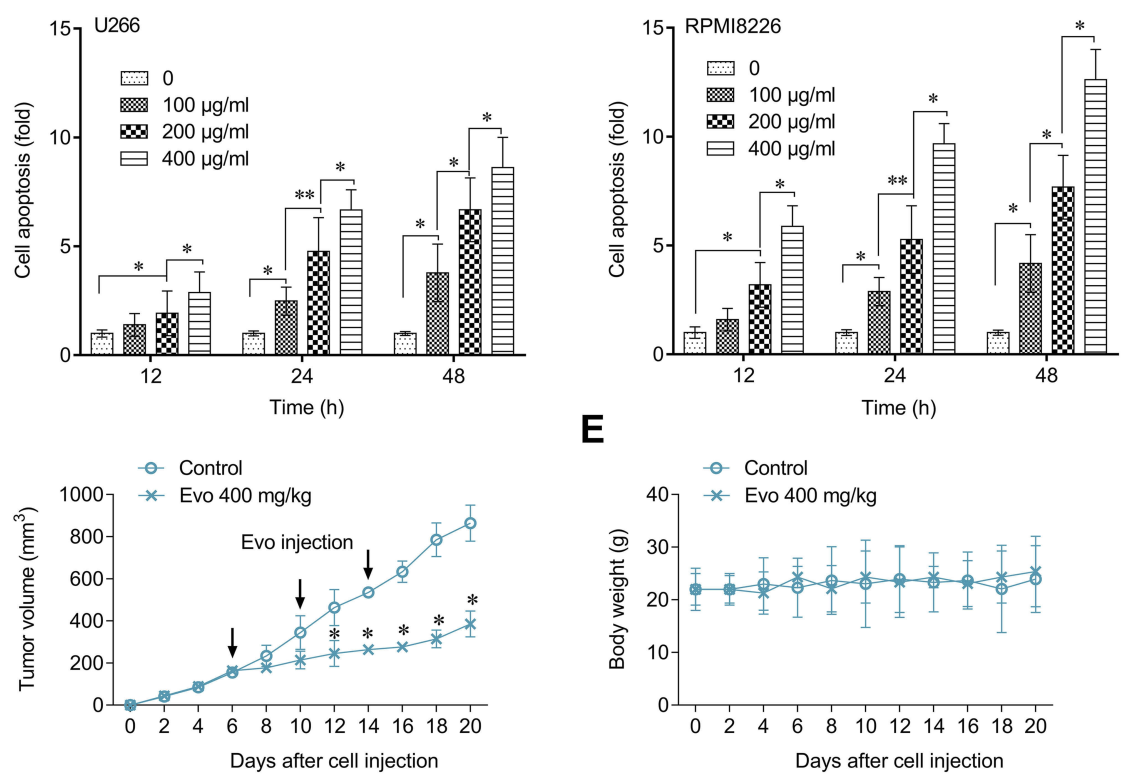

E

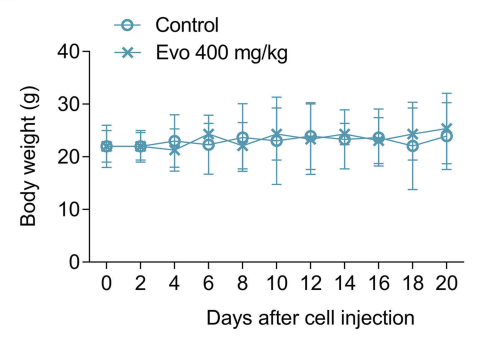

$\mathbf{F}$

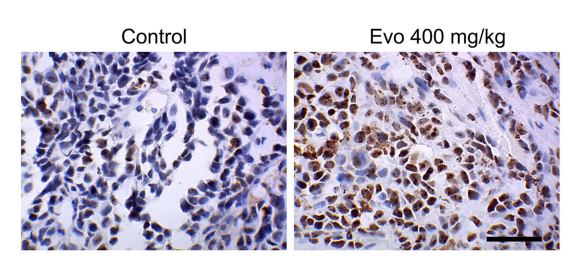

G
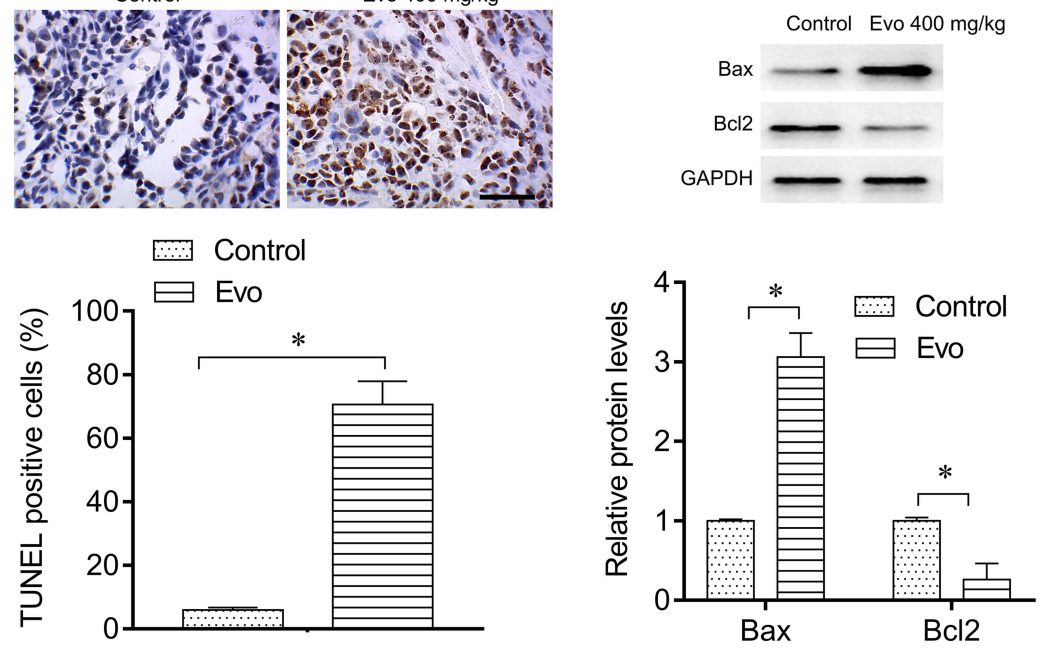

Figure I Evo treatment suppresses multiple myeloma cell growth in vitro and in vivo. (A) Cell viability of MM cells and healthy B lymphocytes (HBC) isolated from donor after various concentrations of Evo treatment for 24 hrs. Data were normalized to the untreated group. (B) Cell survival of U266, RPMI8226 or HBC after Evo treatment $(400 \mu \mathrm{g} / \mathrm{mL})$ for the indicated time. (C) Cell apoptosis in U266 and RPMI8226 cells after indicated treatment. Data were normalized to the untreated group. (D) Xenograft tumor volume change after indicated treatment. (E) Distribution of mice bodyweight during treatment. (F) IHC detecting the TUNEL expression in Xenograft tumor tissues (upper), and quantification (lower). Scale Bar $=100 \mu \mathrm{m}$. (G) Western blot detecting Bax and Bcl-2 protein expression level in Xenograft tumor (upper), and quantification (lower). *p $<0.05$; **p $<0.0$ I. 

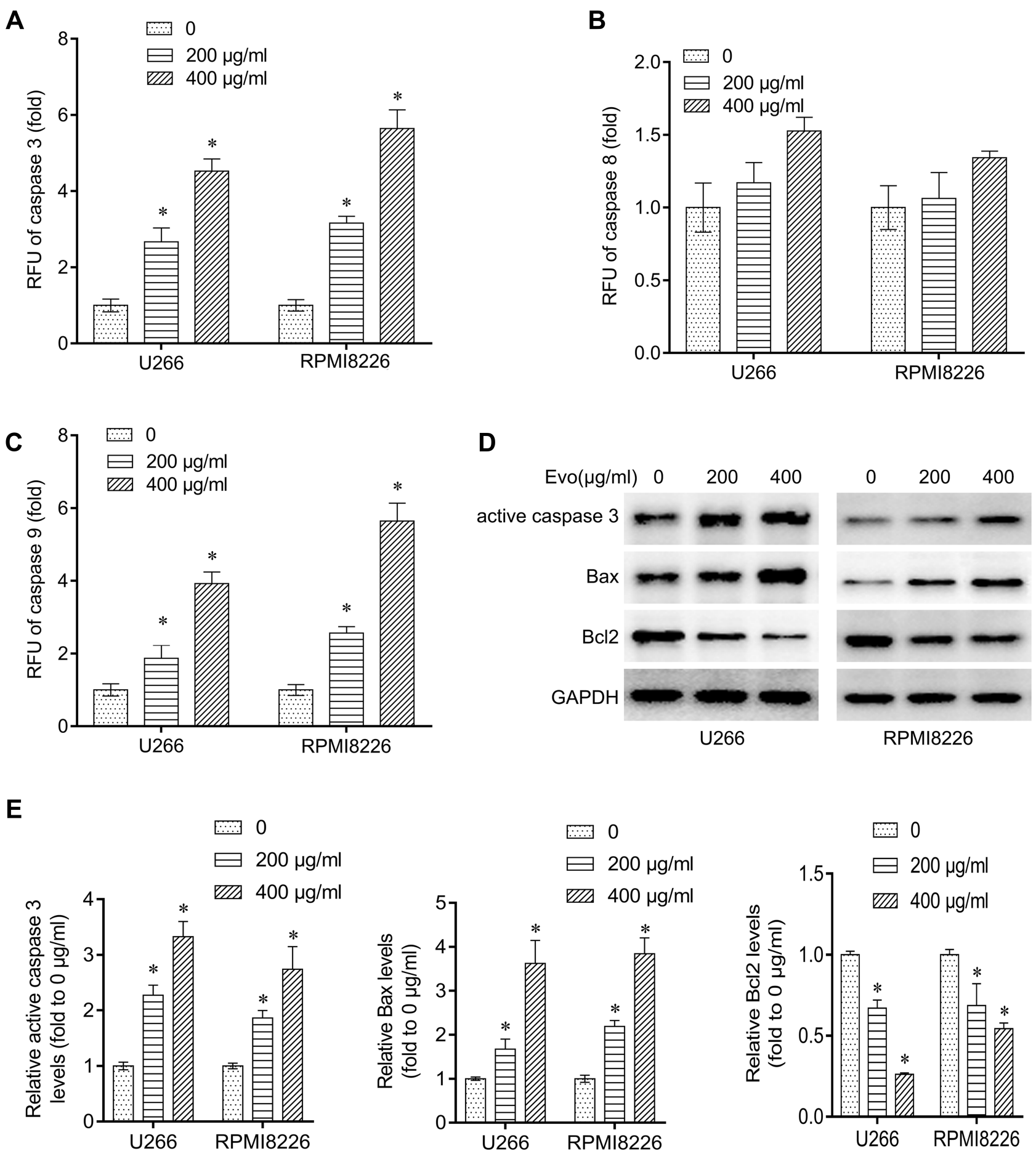

Figure 2 Evo treatment activated the intrinsic apoptosis pathway in multiple myeloma cells. (A-C) Analysis of caspase-9, -8 and -3 activities in U266 and RPMI8226 after EVO treatment $(200,400 \mu \mathrm{g} / \mathrm{mL})$ for $24 \mathrm{hrs}$. Caspase activity was measured by the relative fluorescent units (RFU) of cleaved substrates. (D) Western blot detecting active caspase 3, Bax and $\mathrm{Bcl}-2$ protein expression level in U266 and RPMI8226 after EVO treatment (200, $400 \mu \mathrm{g} / \mathrm{mL})$ for 24 hrs. (E) Quantification of the western bands in (D). *P < 0.05 .

cells with bortezomib alone, or together with Evo, and found a more potently reduction in cell viability and a higher apoptosis than that bortezomib single treatment (Figure 4A and B). In addition, we also confirmed that combination of bortezomib with Evo significantly reduced xenograft tumor volume (Figure 4C). The increased expression of active caspase- 3 and Bax, and the decreased levels of Bcl-2 and Mcl-1 revealed more apoptotic cells in tumor (Figure 4D). These evidences strongly indicated that Evo could enhance bortezomib effects on MM cells. 
A

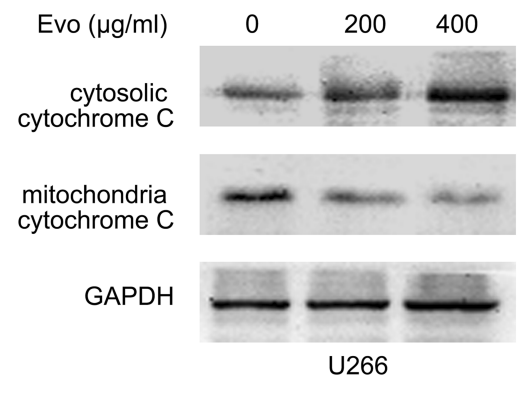

C

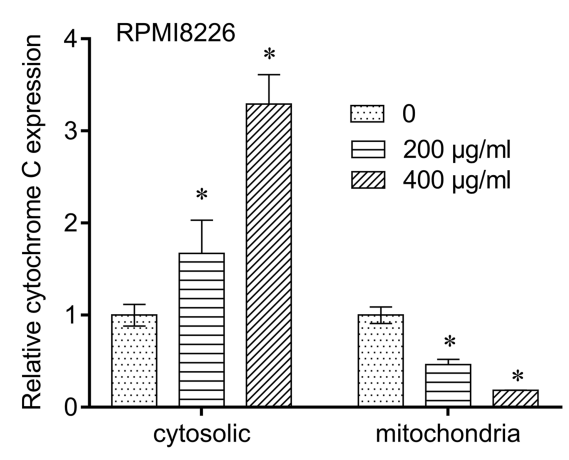

$\mathbf{E}$

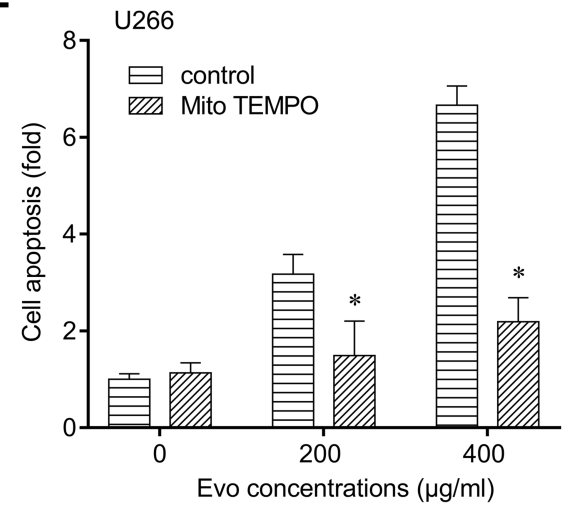

F

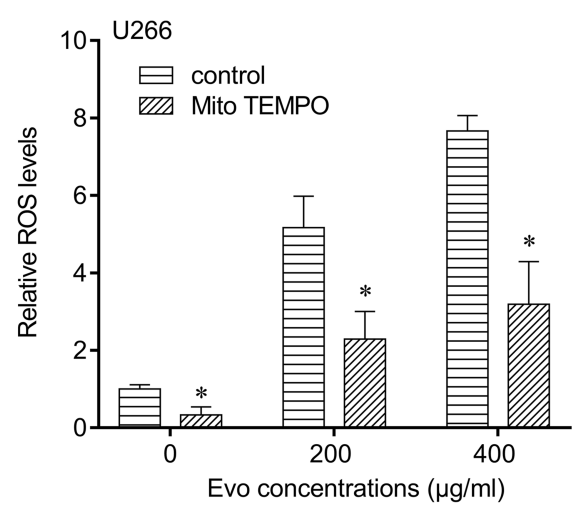

B

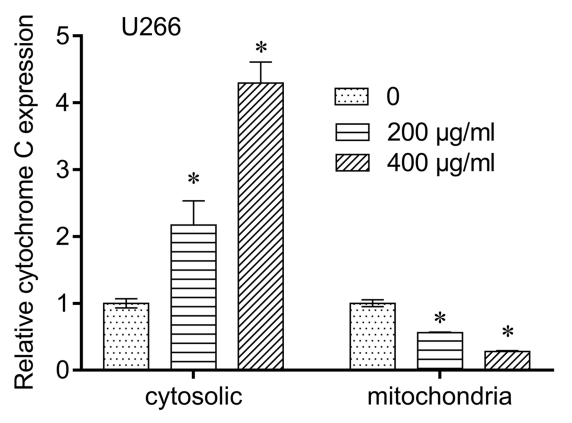

D

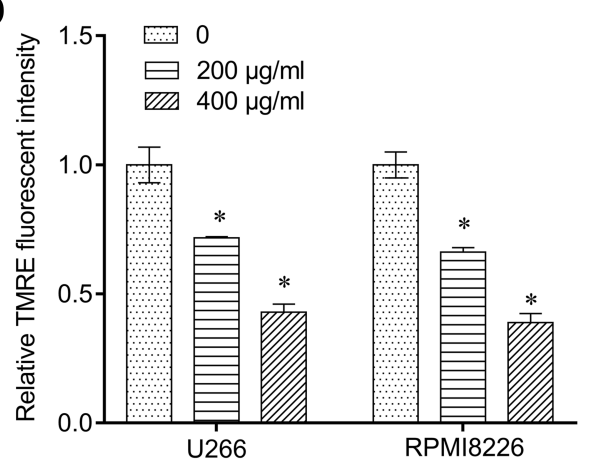

Figure 3 Evo-induced apoptosis was mediated by mitochondria. (A) Cytosolic and mitochondrial cytochrome C expression level in U266 and RPMI8226 after Evo treatment $(200,400 \mu \mathrm{g} / \mathrm{mL})$ for 24 hrs. (B) Quantification of the western bands of cytosolic and mitochondrial cytochrome C expression level in U266 in (A). (C) Quantification of the western bands of cytosolic and mitochondrial cytochrome C expression level in RPMI8226 in (A). (D) Mitochondrial membrane potential of U266 and RPMI8226 after Evo treatment (200, $400 \mu \mathrm{g} / \mathrm{mL})$ for $24 \mathrm{hrs}$. (E) Cells were treated with Evo $(200,400 \mu \mathrm{g} / \mathrm{mL})$ for $24 \mathrm{hrs}$ with or without $20 \mu M$ MitoTEMPO. Cell apoptosis was determined after treatment in two U266 and RPMI8226 cells. (F) ROS production was determined after treatment in two U266 and RPMI8226 cells. *P < 0.05. 
A

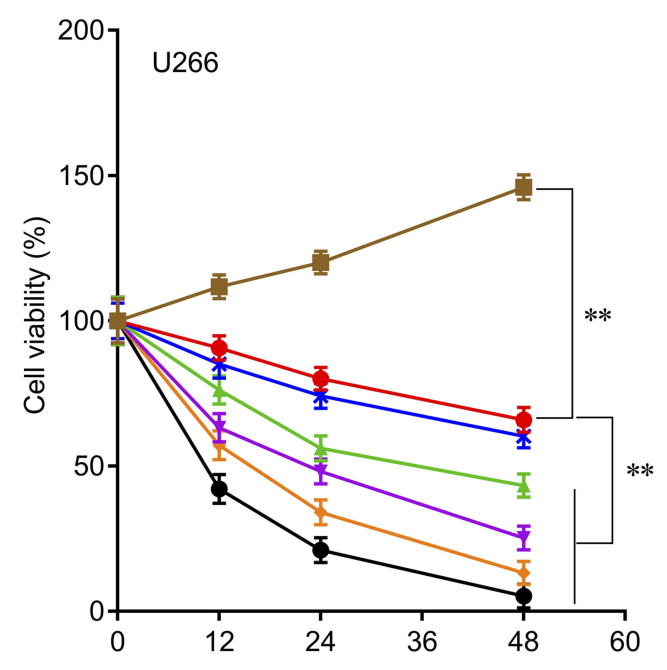

\section{B}

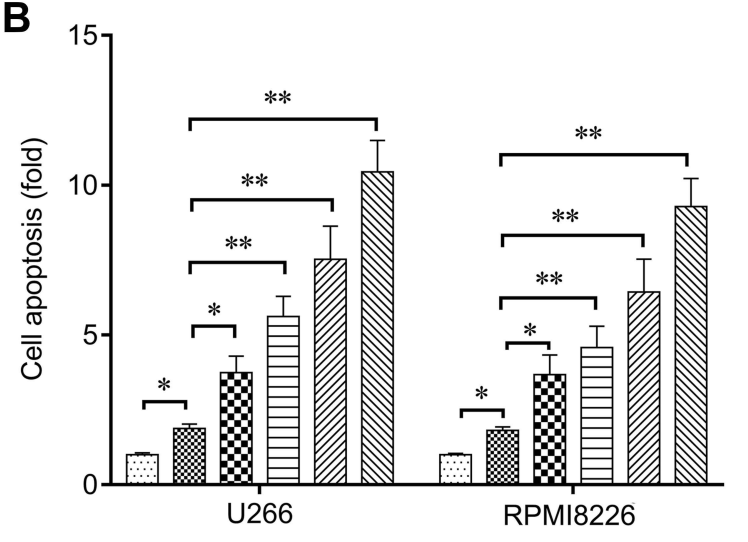

․․ Contro

플 Bort

80 Bort+50

$\boxminus$ Bort+100

U2] Bort+200

Bort+400

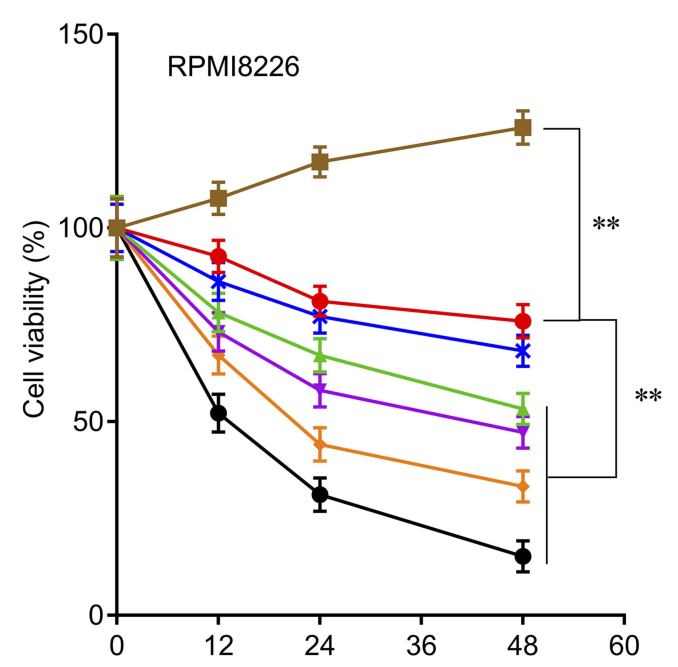

C

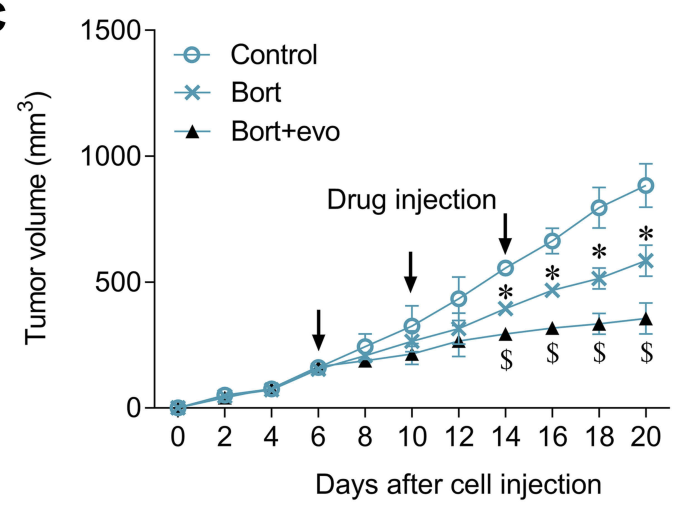

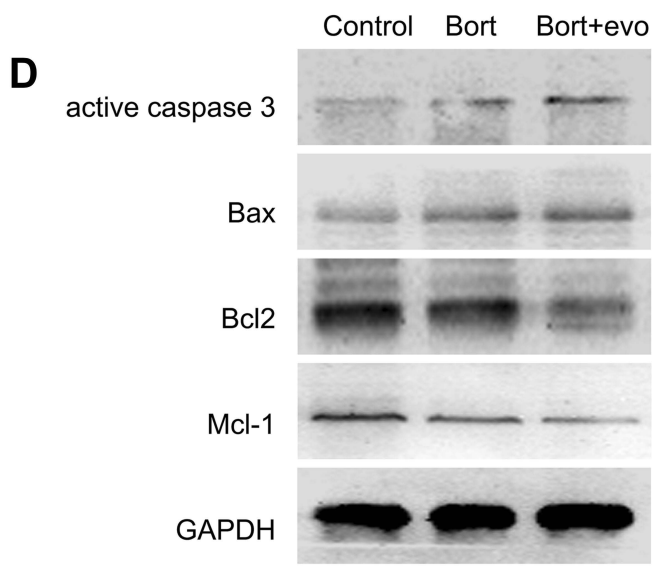

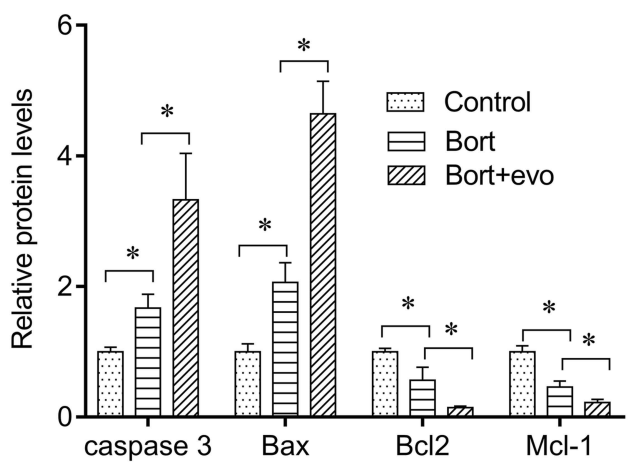

Figure 4 Evo synergized with bortezomib against multiple myeloma. (A) Cells were treated with bortezomib (Bort, 40 nM) or con-treated with Evo at indicated concentrations. Cell viability in U266 and RPMI8226 cells after indicated treatment for 24 hrs. (B) Cell apoptosis in U266 and RPMI8226 cells after indicated treatment for $24 \mathrm{hrs}$. (C) xenograft tumor-bearing mice were injected with bortezomib $(50 \mathrm{mg} / \mathrm{kg})$ or co-injected with Evo $(100 \mathrm{mg} / \mathrm{kg})$. Xenograft tumor volume change after indicated treatment. (D) Western blot detecting active caspase-3, Bax, Bcl-2, and MCL-I protein expression level in Xenograft tumor and quantification. ${ }^{*} \mathrm{P}<0.05$; ** $<0.0 \mathrm{I}$.

\section{Discussion}

Evodiae Fructus (EF) is listed in the China Pharmacopoeia (2010 edition). EF is the nearly ripe fruits of Evodia rutaecarpa (Juss.) Benth. var. bodinieri (Dode), which has anti-inflammatory, anti-microbial, and anti-hypertensive properties. ${ }^{15}$ A number of researches also reports anticancer activity of Evo in East Asian and Western researchers. Hu reported that evodiamine inhibits hepatocellular 
carcinoma cells growth in vivo and in vitro through a WWOX-dependent pathway and inducing Aktmediated apoptosis. ${ }^{16,17}$ Chen revealed that Evo inhibits the ovarian cancer cells viability through disruption of mitochondrial membrane potential. ${ }^{18}$ Previous studies indicate that Evo can suppress various cancer cell growth, such as lung cancer, oral cancer, and urothelial cell carcinoma, which is associated with the ROS accumulation and disruption of mitochondrial membrane potential. ${ }^{19-21}$ In hematopoietic malignances, Evo also exhibits anti-cancer effect on K562, U937 cells, and T-lymphocytes, ${ }^{22-24}$ but the effects and underlying mechanisms on MM remain unknown. In this study, we at the first time investigate the anti-cancer activity of Evo on MM cells. We found that Evo selectively inhibits MM cell growth in both time and dose-dependent manner.

ROS accumulation is a stress signal, which can cause mitochondrial membrane permeabilization, leading to disruption of mitochondrial membrane potential. This disruption leads to activation of intrinsic apoptosis pathway after cytochrome $\mathrm{C}$ release into the cytosol. ${ }^{25}$ In the present study, we demonstrated that Evo induced apoptosis through the intrinsic apoptosis pathway in MM cells by increasing ROS production.

Malignant cells generally produce more mitochondria ROS than non-malignant cells to maintain redox homeostasis. In addition, antioxidant molecules and thioredoxin reductase are upregulated in MM, which is correlated with tumor proliferation and chemoresistance. ${ }^{26}$ Inhibition of thioredoxin reductase significantly inhibited myeloma cell proliferation by regulating HO-1 in a ROS-dependent manner via the Nrf2 signaling pathway. These findings suggest that combination targeting multiple antioxidant systems with other therapeutics will improve therapeutic effectives for patients with $\mathrm{MM}^{27}$ Previous study demonstrated that the bortezomib-induced apoptosis was via thioredoxin reductase inhibition; rescue of thioredoxin reductase 2 conferred MM cell resistance to bortezomib accompany with ROS accumulation decrease. ${ }^{28}$ Bortezomib, a proteasome inhibitor, prolongs activation of ER stress signaling and increased ROS production, finally leading to cell apoptosis. ${ }^{29}$ In this study, we revealed that Evo enhanced the effects of Bortezomib on inhibiting MM cell growth. However, we are not clear the pathway that Evo achieved these effects. Further investigation needs to illustrate these questions.

In summary, we provide evidence that Evo has selective cytotoxicity in MM cells based on its mitochondrial
ROS-inducing activity. Application of Evo and bortezomib might enhance the anti-cancer effect on MM cells.

\section{Author Contributions}

All authors contributed to data analysis, drafting and revising the article, gave final approval of the version to be published, and agree to be accountable for all aspects of the work.

\section{Ethics Approval and Informed Consent}

The blood donors have provided written informed consent. This study was approved by the Ethical Review committee of the Third Xiangya Hospital, Central South University (Changsha, China). Animal experiments were approved by the Ethical Review committee of the Third Xiangya Hospital following the National Research Council's Guide for the Care and Use of Laboratory Animals (Eight Edition).

\section{Disclosure}

The authors report no funding and no conflicts of interest in this work.

\section{References}

1. Gerecke C, Fuhrmann S, Strifler S, et al. The diagnosis and treatment of multiple myeloma. Dtsch Arztebl Int. 2016;113(27-28):470-476. doi:10.3238/arztebl.2016.0470

2. Landgren O. Shall we treat smoldering multiple myeloma in the near future? Hematol Am Soc Hematol Educ Program. 2017;2017 (1):194-204. doi:10.1182/asheducation-2017.1.194

3. Rajkumar SV. Multiple myeloma: 2016 update on diagnosis, risk-stratification, and management. Am J Hematol. 2016;91 (7):719-734. doi:10.1002/ajh.24402

4. Muchtar E, Magen H, Gertz MA. High-risk multiple myeloma: a multifaceted entity, multiple therapeutic challenges. Leuk Lymphoma. 2017;58(6):1283-1296. doi:10.1080/10428194.2016.1233540

5. Yin Z, Yang J, Ning R, et al. Signal pathways, diseases, and functions associated with the miR-19a/92a cluster and the use of berberine to modulate the expression of this cluster in multiple myeloma cells. J Biochem Mol Toxicol. 2018;32(6):e22057. doi:10.1002/jbt.2018.32. issue-6

6. Peng X, Zhang Q, Zeng Y, et al. Evodiamine inhibits the migration and invasion of nasopharyngeal carcinoma cells in vitro via repressing MMP-2 expression. Cancer Chemother Pharmacol. 2015;76 (6):1173-1184. doi:10.1007/s00280-015-2902-9

7. Qiu C, Gao LN, Yan K, Cui YL, Zhang Y. A promising antitumor activity of evodiamine incorporated in hydroxypropyl-betacyclodextrin: pro-apoptotic activity in human hepatoma HepG2 cells. Chem Cent J. 2016;10:46. doi:10.1186/s13065-016-0191-y

8. Zhang Y, Wu LJ, Tashiro S, Onodera S, Ikejima T. Evodiamine induces tumor cell death through different pathways: apoptosis and necrosis. Acta Pharmacol Sin. 2004;25(1):83-89.

9. Li YL, Zhang NY, Hu X, et al. Evodiamine induces apoptosis and promotes hepatocellular carcinoma cell death induced by vorinostat via downregulating HIF-1alpha under hypoxia. Biochem Biophys Res Commun. 2018;498(3):481-486. doi:10.1016/j.bbrc.2018.03.004 
10. Sui H, Zhou LH, Zhang YL, et al. Evodiamine suppresses ABCG2 mediated drug resistance by inhibiting p50/p65 NF-kappaB pathway in colorectal cancer. $J$ Cell Biochem. 2016;117(6):1471-1481. doi: $10.1002 / \mathrm{jcb} .25451$

11. Guo Q, Liu Y, Zhao J, et al. Evodiamine inactivates NF-kappaB and potentiates the antitumor effects of gemcitabine on tongue cancer both in vitro and in vivo. Onco Targets Ther. 2019;12:257-267. doi:10.2147/OTT.S181062

12. Wang R, Deng D, Shao N, et al. Evodiamine activates cellular apoptosis through suppressing PI3K/AKT and activating MAPK in glioma. Onco Targets Ther. 2018;11:1183-1192. doi:10.2147/OTT

13. Liu H, Huang C, Wu L, Wen B. Effect of evodiamine and berberine on miR-429 as an oncogene in human colorectal cancer. Onco Targets Ther. 2016;9:4121-4127. doi:10.2147/OTT.S104729

14. Wang C, Li S, Wang MW. Evodiamine-induced human melanoma A375-S2 cell death was mediated by PI3K/Akt/caspase and Fas-L/NFkappaB signaling pathways and augmented by ubiquitin-proteasome inhibition. Toxicol in Vitro. 2010;24(3):898-904. doi:10.1016/j.tiv. 2009.11.019

15. Yu H, Jin H, Gong W, Wang Z, Liang H. Pharmacological actions of multi-target-directed evodiamine. Molecules. 2013;18(2):1826-1843. doi: $10.3390 /$ molecules 18021826

16. Hu CY, Wu HT, Su YC, et al. Evodiamine exerts an anti-hepatocellular carcinoma activity through a WWOX-dependent pathway. Molecules. 2017;22(7):1175. doi:10.3390/molecules22071175

17. Yang F, Shi L, Liang T, et al. Anti-tumor effect of evodiamine by inducing Akt-mediated apoptosis in hepatocellular carcinoma. Biochem Biophys Res Commun. 2017;485(1):54-61. doi:10.1016/j. bbrc.2017.02.017

18. Chen TC, Chien CC, Wu MS, Chen YC. Evodiamine from Evodia rutaecarpa induces apoptosis via activation of JNK and PERK in human ovarian cancer cells. Phytomedicine. 2016;23(1):68-78. doi:10.1016/j.phymed.2015.12.003

19. Sachita K, Kim Y, Yu HJ, Cho SD, Lee JS. In vitro assessment of the anticancer potential of evodiamine in human oral cancer cell lines. Phytother Res. 2015;29(8):1145-1151. doi:10.1002/ptr.5359
20. Mohan V, Agarwal R, Singh RP. A novel alkaloid, evodiamine causes nuclear localization of cytochrome-c and induces apoptosis independent of p53 in human lung cancer cells. Biochem Biophys Res Commun. 2016;477(4):1065-1071. doi:10.1016/j.bbrc.2016.07.037

21. Shi CS, Li JM, Chin CC, et al. Evodiamine induces cell growth arrest, apoptosis and suppresses tumorigenesis in human urothelial cell carcinoma cells. Anticancer Res. 2017;37(3):1149-1159.

22. Huang YC, Guh JH, Teng CM. Induction of mitotic arrest and apoptosis by evodiamine in human leukemic T-lymphocytes. Life Sci. 2004;75(1):35-49. doi:10.1016/j.lfs.2003.11.025

23. Sun C, Zhang G, Luan S, et al. Evodiamine inhibits the proliferation of leukemia cell line K562 by regulating peroxisome proliferators-activated receptor gamma (PPARgamma) pathway. J Recept Signal Transduct Res. 2016;36(4):422-428. doi:10.3109/10799893.2015.1122040

24. Lee TJ, Kim EJ, Kim S, et al. Caspase-dependent and caspase-independent apoptosis induced by evodiamine in human leukemic U937 cells. Mol Cancer Ther. 2006;5(9):2398-2407. doi:10.1158/1535-7163.MCT-06-0167

25. Kalkavan H, Green DR. MOMP, cell suicide as a BCL-2 family business. Cell Death Differ. 2018;25(1):46-55. doi:10.1038/cdd.2017.179

26. Raninga PV, Di Trapani G, Vuckovic S, Tonissen KF. Cross-talk between two antioxidants, thioredoxin reductase and heme oxygenase-1, and therapeutic implications for multiple myeloma. Redox Biol. 2016;8:175-185. doi:10.1016/j.redox.2016.01.007

27. Song IS, Kim HK, Lee SR, et al. Mitochondrial modulation decreases the bortezomib-resistance in multiple myeloma cells. Int $J$ Cancer. 2013;133(6):1357-1367. doi:10.1002/ijc.v133.6

28. Fink EE, Mannava S, Bagati A, et al. Mitochondrial thioredoxin reductase regulates major cytotoxicity pathways of proteasome inhibitors in multiple myeloma cells. Leukemia. 2016;30(1):104-111. doi:10.1038/leu.2015.190

29. Santos CX, Tanaka LY, Wosniak J, Laurindo FR. Mechanisms and implications of reactive oxygen species generation during the unfolded protein response: roles of endoplasmic reticulum oxidoreductases, mitochondrial electron transport, and NADPH oxidase. Antioxid Redox Signal. 2009;11(10):2409-2427. doi:10.1089/ars.2009.2625
OncoTargets and Therapy

\section{Publish your work in this journal}

OncoTargets and Therapy is an international, peer-reviewed, open access journal focusing on the pathological basis of all cancers, potential targets for therapy and treatment protocols employed to improve the management of cancer patients. The journal also focuses on the impact of management programs and new therapeutic

Submit your manuscript here: https://www.dovepress.com/oncotargets-and-therapy-journa agents and protocols on patient perspectives such as quality of life, adherence and satisfaction. The manuscript management system is completely online and includes a very quick and fair peer-review system, which is all easy to use. Visit http://www.dovepress.com/ testimonials.php to read real quotes from published authors. 